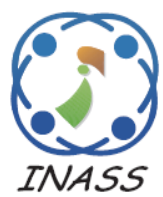

\title{
Efficient Channel Estimation and Signal Detection Schemes for Multiple Input and Multiple Output System under WiMax Environment
}

\author{
KVN Kavitha ${ }^{1 *} \quad$ Kala Praveen Bagadi $^{2}$ \\ ${ }^{1,2}$ School of Electronics Engineering, VIT University, Vellore, India \\ * Corresponding author’s Email: kvnkavitha@yahoo.co.in
}

\begin{abstract}
The multiple input Multiple Output (MIMO) is a wireless technology that can offer high spectral efficiency, capacity gain and/or diversity gain. On the other hand, the Worldwide Interoperability for Microwave Access (WiMAX) is a promising wireless technology which delivers data at high rates covering larger area. To retrieve desired signals in such technologies, we need to incorporate appropriate Channel estimation and signal detection techniques at the receiver. Thus, in this paper we propose Scaled Least Square-Maximum Likelihood (SLS-ML) and Reduced Minimum Mean Square Error-ML (RMMSE-ML) receivers for MIMO system under WiMAX environment. The proposed techniques are consistently outperforming the exiting Least Square-Maximum Likelihood (LS-ML) and Minimum Mean Square Error-ML (MMSE-ML) receivers. The RMMSE and SLS techniques minimize channel estimation error and the Maximum Likelihood (ML) detector cancels residual interference from other transmitting antennas. In the simulation study, the Bit Error Rate (BER) performance evaluation of $2 \times 2$ and $4 \times 4$ antenna structures with different detector and channel estimator techniques over various Erceg's channel models has been discussed. The investigations show that the ML detection applied to the mobile WiMAX systems provide better performance than other detector systems, irrespective of the number of antenna array size and the fading effect.
\end{abstract}

Keywords: MIMO, WiMAX, STBC, RMMSE, SLS, ML detector.

\section{Introduction}

The demand for communication system bandwidth is rising day by day. A wireless system with high spectral efficiency is needed to transmit data with high speed. But the fading of signals during transmission reduces this efficiency. To overcome the problems involved in the transmission of data and to satisfy the rising demand of efficient communication systems, there is a need to develop new modulation techniques to meet the demand. These requirements cannot be delivered using the traditional techniques.

The last few years have seen the development of Orthogonal Frequency Division Multiplexing (OFDM) as a multicarrier signal modulation technique for digital communication environment with high speed data transmission. It is widely used over the frequency selective channels due to its robust nature and its capability to maintain a constant channel characteristic and to bring down the operational complexity of the receiver [1]. The focus is also on multiple input-multiple output (MIMO) system which has the capability to exploit multipath propagation. The MIMO systems give higher bandwidth efficiency and improved performance compared to the traditional single antenna systems [2]. Since OFDM and MIMO have their own merits, this combination can become promising solution for next generation wireless system [3-6]. Hence, the MIMO-OFDM technique has become a major tool in the communication systems for Worldwide Interoperability for Microwave Access (WiMAX) [7, 8]. WiMax is a wireless access technology standard designed to offer greater wireless coverage over long distances, from point to multi-point networks [9, 10]. As pointed out by Shannon [11], the essential task in 
communication is to reproduce a message selected at one point, exactly or approximately at another point.

At the receiver of the MIMO-OFDM, the channel estimation and signal detection are the two major issues need to be addressed properly. The channel state information can be obtained through training, blind and semi blind channel estimation techniques. The blind channel estimation is based on the statistical information of the channel and certain properties of the transmitted signals [12, 13]. These techniques are applicable to slow time-varying channels due to its need for a long data record. The training-based channel estimation is based on the training data (pilots) sent from the transmitter that is known $a$ priori at the receiver $[14,15]$. In general, the mobile wireless applications are fast timevarying and hence the training based channel estimation is a preferable one. Further, the semiblind channel techniques are hybrid of blind and training estimation techniques, which utilizes pilots and other natural constraints to perform channel estimation. In the training based techniques, the classical Least Square (LS) estimator does not require any channel statistics, but they are affected by mean square error in a huge amount [16]. To overcome this limitation, the estimator is scaled, improved and named as Scaled LS (SLS) estimator. Similarly the classical The Minimum Mean Square Error (MMSE) estimator requires perfect knowledge of the correlation matrix assumption [16]. This assumption is not reachable in practical situations. Thus, the Relaxed MMSE (RMMSE) algorithm can be used as it requires only small amount of channel correlation matrix. Once channel state information is known, the transmitted signals can be estimated through Signal detection problem [17, 18]. Out of several detectors, though the MMSE one is low complex, it is suffering from limited performance. The Maximum Likelihood (ML) detector may give optimal performance, but its complexity increases exponentially with number of transmitting antennas. Thus, this paper aims at identifying a MIMO receiver with both channel estimation and signal detection. The performance of the MIMO receiver is validated in WiMAX Environment.

The rest of the paper is organized as follows. The multi antenna channel model is presented in Section 2. Section 3 describes about channel estimation and overview of detection techniques are given in Section 4. Linear and optimum signal detection techniques are discussed in Section 5 and Section 6 respectively. Simulation analysis with results is elaborated in Section 7. Finally, the conclusion is presented in Section 8.

\section{Multi antenna channel model}

In a wireless communication system with multiple antennae, the channels that transmit data can be considered as linear and time invariant model. Mathematically, the received signal is represented in Eq. (1) as,

$\boldsymbol{y}=\boldsymbol{H s}+\boldsymbol{w}$

In Eq.(1), $\boldsymbol{H}$ is a $N_{t} \times N_{r}$ complex valued channel whose realization is known to the receiver through estimation processing, $s$ is the transmitted symbol with $N_{t-}$ dimension, and $\boldsymbol{w}$ is a Gaussian noise with zero mean and $\sigma^{2}$ dispersion around the mean value.

The major objective of a MIMO detection system is to estimate the transmitted input signal vector based on the channel information available at the receiver and signal vector collected at the receiver end. A transmitted symbol estimate $\hat{\mathbf{s}}$, is measured by the detector at the receiving end of the system. The optimal detector is the one which reduces the average error probability $(\hat{\boldsymbol{s}} \neq \boldsymbol{s})$.

\section{Channel estimation}

In coherent detection process, information about the channel is required to mitigate the fading effects appears in a wireless environment caused by the channel. The channel estimation in a multiple antennae system is a primary as well as challenging task too. In this, signals are received as combinations of the multiple signals from transmit antennas. Initially classical Training based estimation algorithms has been used and their performances are compared in terms of bit error rate over various SUI channel model. Finally some modifications are made in the basic algorithm, in order to reduce the error rate.

\subsection{Scaled least square (sls) estimation}

The SLS estimation of the channel can be expressed as,

$\widehat{H}_{S L S}=\gamma_{o} \widehat{H}_{L S}=\frac{\operatorname{tr}\left\{R_{H}\right\}}{\sigma^{2} N_{r} \operatorname{tr}\left\{\left(S S^{H}\right)^{-1}\right\}+\operatorname{tr}\left\{R_{H}\right\}} R S^{\dagger}$

$N_{r}$ - Number of receiving antennas; $R_{H}=E\left\{H^{H} H\right\}-$ channel correlation matrix; Receive noise power $-\sigma^{2}$. To further reduce the MSE, the MMSE estimator makes use of the channel's second order statistics. The linear MMSE gives better performance at small SNR compared to that of LS, but at the expense of increase in complexity. 


\subsection{Relaxed minimum mean square error (rmmse) estimation}

The MMSE channel estimate in the frequency domain is,

$H_{M M S E}=Y \times A$

The matrix $A$ is calculated as,

$A=\arg \min _{A} E\left\{\|H-Y A\|_{F}^{2}\right\}$

The optimal value of $A$ can be obtained from $\partial \varepsilon / \partial A=0$ and is expressed by

$A=\left(S^{H} R_{H} S+\sigma^{2} N_{r} I\right)^{-1} S^{H} R_{H}$

Hence, the linear MMSE estimator can be represented as

$H_{M M S E}=Y\left(S^{H} R_{H} S+\sigma^{2} N_{r} I\right)^{-1} S^{H} R_{H}$

$R_{H}$ - Covariance matrix of the channel with a variance of $\sigma^{2}$ and $Y$ is the received signal. The covariance matrix of the channel requirement is the drawback of this method. But in case of mobile environment, channel features are not constant. Due to this it is difficult to track the covariance matrix. In RMMSE, only small amount of channel correlation matrix is sufficient. The MMSE requires perfect knowledge of the correlation matrix assumption. This assumption is not reachable in practical situations. It can be alternatively written as $\alpha I$ in lieu of $R_{H}$. To minimize the error, the parameter $\alpha$ can be adjusted. Replacing $R_{H}$ with $\alpha I$ and applying the matrix inversion lemma, we can rewrite this equation as,

$\widehat{H}=\alpha Y\left(\alpha S^{H} S+\sigma^{2} N_{r} I\right)^{-1} S^{H}$

By assuming orthogonal training, the channel MSE can be computed as

$\mathcal{J}_{R M M S E}=\left(E\left\{\|H-\widehat{H}\|_{F}^{2}\right\}\right)$

MSE can be minimized by substituting optimum value of $\alpha$. If $\sigma^{2}>0$, then $\mathcal{J}_{R M M S E}<\mathcal{J}_{L S}$ and hence the relaxed MMSE channel estimation method operates always greater than the LS estimator. The enhancement of the RMMSE estimator over the LS estimator is notably prominent if the SNR is low. Performance of all these estimators have been studied under WiMAX scenario and tested over SUI channel model.

\section{Overview of detection methods}

The basic research in MIMO technology started in early 1960's. Shnidman contributed to the MIMO detection technology in 1967 [19] by considering an equalization problem of a pulse modulation technique with limited bandwidth. As compared to the single-input and single-output systems, the MIMO works on the principle of diversity, where multiple symbols are transmitted concurrently and the receiver is provided with multiple forms of the arrived signal. The receiver detects and decodes the symbols based on the amount interference and random noise that affects the quality of the transmitting messages as shown in Fig.1. The diversity helps to reduce the error rate by stabilizing the transmitted and received data.

The channel estimation and signal detection are required to be performed at the receiver end for intelligible recovery of transmitted data. The detection of symbol may be done individually or jointly. In joint symbol detection, a symbol is detected by considering the characteristics of the other symbols in addition with the current symbol features.

Thus the joint detection system gives a better performance compared to the other one mentioned, even though more computational complexity is involved. The joint detection of multiple symbols is one of the major advantages of the various MIMO techniques. The limiting characteristic to the benefits of the MIMO systems is the occurrence of co-channel interference (CCI), which is generally encountered in these systems. The complexity of the common algorithms perceived for optimal solution of the problem increases when the number of decision variables involved is more. This fact has been observed in the MIMO detection algorithms based on maximum a posteriori (MAP) criterion as well as Maximum Likelihood criterion.

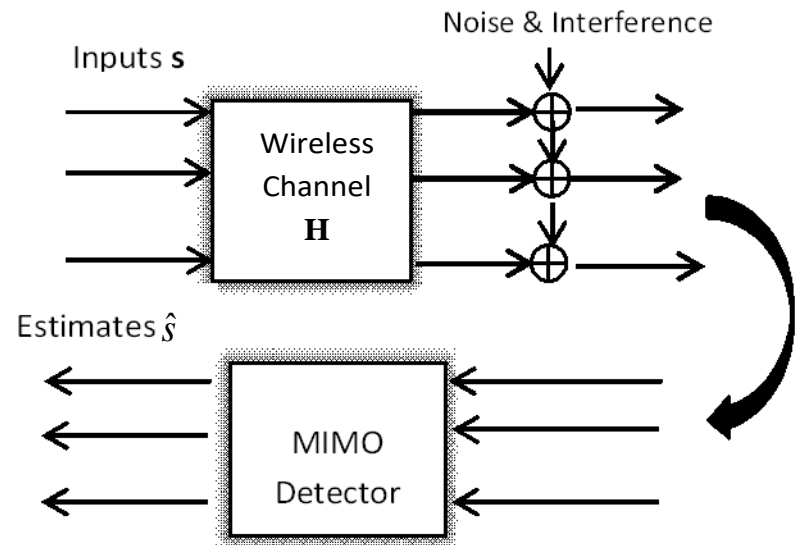

Figure. 1 MIMO Detection 
The detection schemes can be coherent or noncoherent. The detection of symbol $s$ is considered to be coherent, if the instantaneous value of the channel matrix $\boldsymbol{H}$ is established explicitly through channel estimation. On the other hand, if the explicit estimation of the channel is evaded the detection of symbol $s$ belongs to non-coherent scheme. The channel estimation in the latter scheme is done implicitly or it is completely avoided. The signal detection is performed by using the statistical information of the channel matrix $\boldsymbol{H}$.

In a multiple symbol differential detection [20] [21], the input symbols are encoded through differential encoding. The detection is performed by studying the symbols block by block in sequence. But this increases the computational complexity as compared to the symbol-by-symbol detection performed in coherent MIMO systems. Also in noncoherent detectors, due to the exponential increase in computation, the power efficiency degrades, leading to the performance loss in the system. To counteract this performance loss in the system, the block size should be sufficiently large in these detector systems. Therefore, a trade-off between the required performance and the amount of complexity is necessary to choose an optimum block size.

\section{Linear mimo signal detectors}

When the estimation of the symbols is based on linear transformation of the output signal vector, the detectors are known as linear MIMO detectors. The algorithm shows low complexity but with significant loss in performance metrics. The decision statistics of linear MIMO detectors is expressed as,

$$
d=\boldsymbol{F} \boldsymbol{y}
$$

In Eq. (5), $\boldsymbol{F}$ is the linear transformation matrix. The concept of linear MIMO detectors is depicted in Fig. 2. Different types of linear detectors used in MIMO have been discussed below with the corresponding decision metric. The Matched Filter (MF) is a kind of linear detector having the lowest complexity in computing.

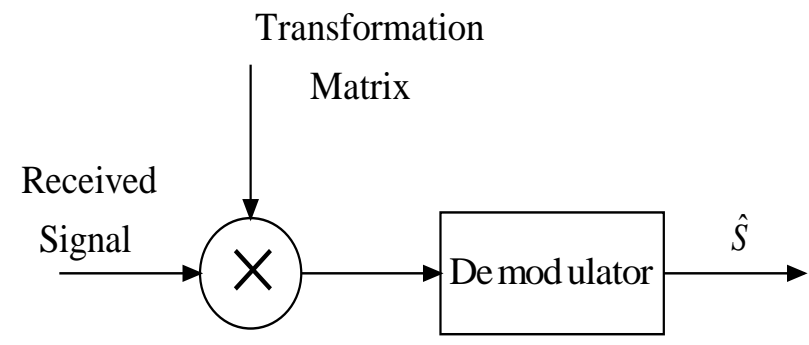

Figure. 2 Linear Detector
The linear transformation matrix of the MF detector is expressed as,

$\boldsymbol{F}_{M F}=\boldsymbol{H}^{H}$

Upon using the transformation matrix or filtering matrix definition, the decision statistics of the MF detector becomes,

$d=\boldsymbol{F}_{M F} \boldsymbol{y}=\boldsymbol{H}^{H} \boldsymbol{H} \boldsymbol{s}+\boldsymbol{H}^{H} \boldsymbol{w}$

The Zero Forcing (ZF) detector is another kind of linear transformation based detector developed by Van Etten [22] for a multiple channel multiplexing communication system exposed to both inferences namely, Inter Symbol Interference and interchannel interference. The detector is first analysed in the space division multiplexing based VBLAST systems by Foschini, Golden, Wolniansky and Valenzuela [23-25] after the arrival of multipleantenna technologies during mid-1990's.

The transmitted signal is detected from the least square error as follows in Eq. (12) and Eq. (13),

$$
\begin{aligned}
& \boldsymbol{y}-\boldsymbol{H} \boldsymbol{s}^{2}=(\boldsymbol{y}-\boldsymbol{H} \boldsymbol{s})^{H}(\boldsymbol{y}-\boldsymbol{H} \boldsymbol{s}) \\
& =-2 \boldsymbol{H}^{H} \boldsymbol{y}+2 \boldsymbol{H}^{H} \boldsymbol{H} \boldsymbol{s}
\end{aligned}
$$

The optimal minima of the symbol s can be found from the relation $\partial\|\boldsymbol{y}-\boldsymbol{H} \boldsymbol{s}\|^{2} / \partial s=0$. It becomes,

$-2 \boldsymbol{H}^{H} \boldsymbol{y}+2 \boldsymbol{H}^{H} \boldsymbol{H} \boldsymbol{s}=0$

Therefore, the detected signal is,

$$
\widehat{\boldsymbol{s}}=\left(\boldsymbol{H}^{H} \boldsymbol{H}\right)^{-1} \boldsymbol{H}^{H} \boldsymbol{y}
$$

Now, the linear transformation matrix for the ZF detector can be expressed as,

$\boldsymbol{F}_{Z F}=\left(\boldsymbol{H}^{H} \boldsymbol{H}\right)^{-1} \boldsymbol{H}^{H}(16)$

Therefore, the decision statistics $d$ becomes,

$d=\left(\left(\boldsymbol{H}^{H} \boldsymbol{H}\right)^{-1} \boldsymbol{H}^{H}\right)(\boldsymbol{H} \boldsymbol{s}+\boldsymbol{w})=\boldsymbol{s}+\boldsymbol{F}_{Z F} \boldsymbol{w}$

This signifies complete reduction in interference of the multiple data inputs, even though the noise power is improved.

The earliest linear MIMO detector is the MMSE criterion based detector [26] which was proposed by Shnidman in the year 1967 [19]. The linear transformation matrix of the MMSE criterion is 
designed to reduce the mean square error among the output data and the actual data of the channel. The mean square error between the data is represented as $E\left[|\hat{\boldsymbol{s}}-\boldsymbol{s}|^{2}\right]$, where $\hat{\boldsymbol{s}}$ is the estimate of $\boldsymbol{s}$.

$E\left[|\widehat{\boldsymbol{s}}-\boldsymbol{s}|^{2}\right]=E\left[(\boldsymbol{F} y-\boldsymbol{s})^{H}(\boldsymbol{F} \boldsymbol{y}-\boldsymbol{s})\right]$

The optimal value of the linear transformation matrix $F$ can be calculated from $\partial \mathrm{E}\left[|\hat{\mathbf{s}}-\mathbf{s}|^{2}\right] / \partial \mathrm{F}=$ 0 . Then, the $\boldsymbol{F}$ can be expressed as,

$\boldsymbol{F}=R_{y y}^{-1} R_{y s}$

In Eq. (10), the auto covariance of $y$ is $R_{y y}=$ $E\left[\boldsymbol{y} \boldsymbol{y}^{H}\right]$ and the cross covariance of $\boldsymbol{y}$ and $\boldsymbol{s}$ is defined as $R_{y s}=E\left[y s^{H}\right]$. Therefore,

$R_{y y}=\left(\boldsymbol{H}^{H} \boldsymbol{H}+\sigma^{2} \boldsymbol{I}\right)$

$R_{y s}=\boldsymbol{H}^{H}$

The linear transformation matrix, after replacing $R_{y y}$ and $R_{y s}$ with the above mentioned values, becomes,

$\boldsymbol{F}=\left(\boldsymbol{H}^{H} \boldsymbol{H}+2 \sigma^{2} \boldsymbol{I}\right)^{-1} \boldsymbol{H}^{H}$

Therefore the decision statistics is given by,

$d=\left(\boldsymbol{H}^{H} \boldsymbol{H}+2 \sigma^{2} \boldsymbol{I}\right)^{-1} \boldsymbol{H}^{H} y$

The $\sigma^{2}$ can be neglected for higher values of SNR as it becomes insignificant. Hence, at higher values of SNR the performance of Zero Forcing detector and MMSE detector are almost equal.

At lower SNR values, the performance of linear MMSE detector is found to be better than the linear Zero Forcing detector. The linear MMSE algorithm is able to minimize the total error enforced by the interference between the symbols and the noise, thereby, achieving a good balance between the elimination of interference and enhancement of noise.

\section{Optimum detector and its decision}

The efforts to develop optimum MIMO detector systems started in the year 1976 when Van Etten [27] derived a Maximum likelihood (ML) sequence estimation based receiver for tackling both the interferences ISI and ICI in multiple channel communication systems. Based on certain conditions, the ML receiver is found to approach the performance of an idealized optimum receiver system that is free from both ICI and ISI.
The performance of the receivers are measured based on some important parameters. The minimum error probability criterion is an important parameter in assessing the functioning of the detectors. The maximum a posteriori (MAP) criterion is an optimum MIMO decision criterion which reduces the error probability considering only the observed signals with given set of hypothesis.

The symbol detection problem in a ML Detector can be optimized by formulation the problem as a finite set constrained least squares (LS).

$\hat{\boldsymbol{s}}_{M L}=\arg \left\{\min _{u}\left\|\boldsymbol{y}-\boldsymbol{H} \tilde{\boldsymbol{s}}_{u}\right\|^{2}\right\}, u=1,2, \ldots, 2^{m}$

It is also considered as minimum Euclidean distance (MED) based criterion, where, $\mathrm{m}$ is the number of bits per symbol, $u$ denotes the set of total metric evaluations with respect to the modulation order and $\tilde{s}_{u}$ is the possible transmitted symbol. The optimal detector performs a comprehensive search to find the most probable transmitted signal.

Though, the ML method is an optimal detector, it has huge computational complexity. To solve this weakness, some variations have been included [28, 29].

\section{Simulation outputs and discussion}

The MIMO OFDM system model is simulated using Matlab by considering the parameters of the standard Stanford University Interim (SUI) channel model [30], also known as Erceg's model in WiMAX scenario, along with system parameters presented in Table 1 for different fading conditions. The performance of ML detector is measured and compared with MMSE detector through different channel estimation algorithms. The Scaled Least Square (SLS), Minimum Mean Square Error (MMSE) and Reduced Minimum Mean Square Error (RMMSE) has been applied to track the channel features [16]. Results are tested in two different terrain types (terrain C (SUI-1) and terrain A (SUI-5)) which define various amount of fading effect over the transmitted signal.

Table 1.System Simulation Parameters

\begin{tabular}{|l|l|}
\hline \multicolumn{1}{|c|}{ Parameter } & Specification \\
\hline Open loop MIMO antenna array size & $2 \times 2$ (STBC) \\
\hline Number of OFDM subcarriers & 256 \\
\hline Number of OFDM frames & 100 \\
\hline Number of bits per symbol & 1 \\
\hline Input symbol rate & $1 \mathrm{Mbps}$ \\
\hline
\end{tabular}


Table 2. Erceg's SUI-1 Channel Model Parameters

\begin{tabular}{|l|c|c|c|}
\hline \multirow{2}{*}{ Parameter } & \multicolumn{3}{|c|}{ Specifications } \\
\cline { 2 - 4 } & Path-1 & Path-2 & Path-3 \\
\hline Delay in Path ( $\boldsymbol{\mu s e c})$ & 0 & 0.4 & 0.9 \\
\hline Channel Gain (dB) & 0 & -15 & -20 \\
\hline Doppler Shift $(\mathbf{H z})$ & \multicolumn{3}{|c}{0.5} \\
\hline
\end{tabular}

Table 3. Erceg's SUI-5 Channel Model Parameters

\begin{tabular}{|l|c|c|c|}
\hline \multirow{2}{*}{ Parameter } & \multicolumn{3}{|c|}{ Specifications } \\
\cline { 2 - 4 } & Path-1 & Path-2 & Path-3 \\
\hline Delay in Path ( $\boldsymbol{\mu s e c )}$ & 0 & 4 & 10 \\
\hline Channel Gain (dB) & 0 & -5 & -10 \\
\hline Doppler Shift $(\mathbf{H z})$ & \multicolumn{4}{|c}{2.5} \\
\hline
\end{tabular}

Time domain characteristics of SUI channel with different level of fading is simulated with the specifications mentioned in Table 2 and Table 3.

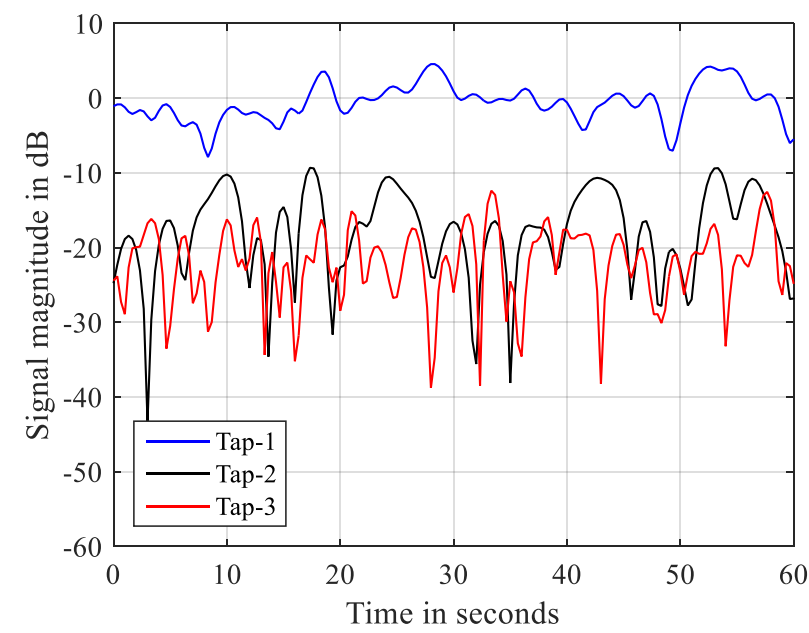

(a)

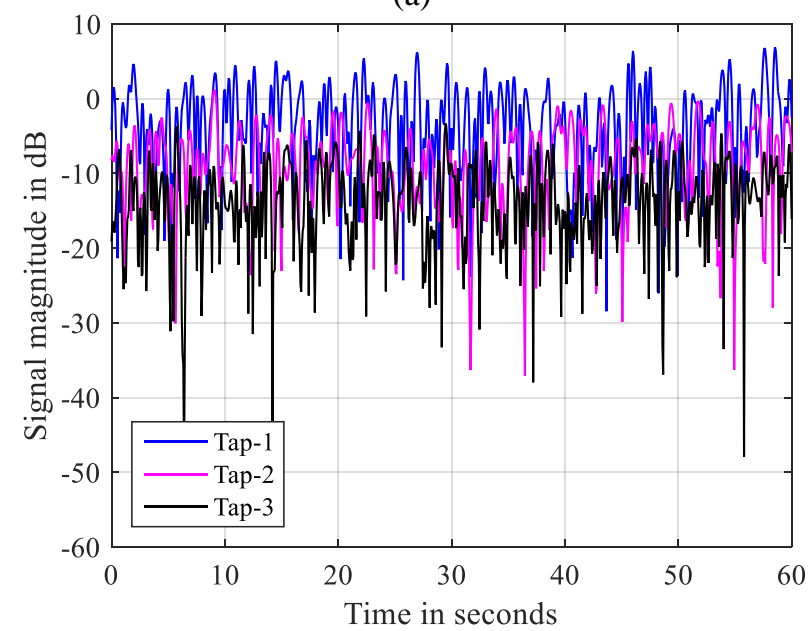

(b)

Figure. 3 Time domain characteristics of (a) SUI-1 (b) SUI-5 channel model

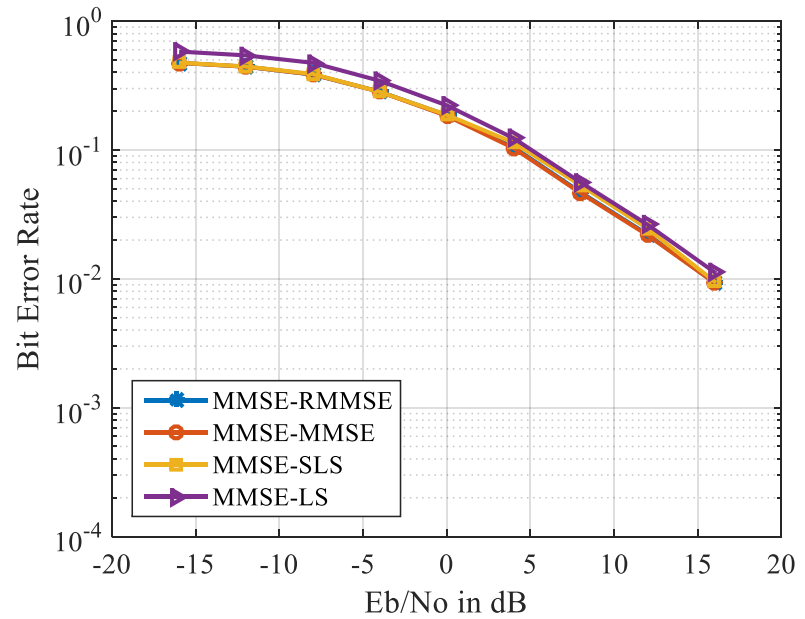

Figure. 4 MMSE detector with various channel estimators over SUI-1channel

The above result in Fig. 3 reflects the fading nature in terms of received signal power for the SUI channel in three different paths over the specified time interval.

The transmitted signal is detected at the receiver end using MMSE algorithm and its error performance is measured for various values of signal to noise ratio with different channel tracking information as shown in Fig. 4. MMSE detector with RMMSE estimated channel gives better error performance at low SNR values than other estimation algorithms. Since, MMSE detector holds the disadvantage of biased estimated output, optimum detector has been selected for the detection signal. The noise power at higher SNR values can be negligible as we seen in eq. (22), and the MMSE detector become ZF detector.

The BER of the ML detector for MIMO system with and without channel tracking is shown in Fig. 5.

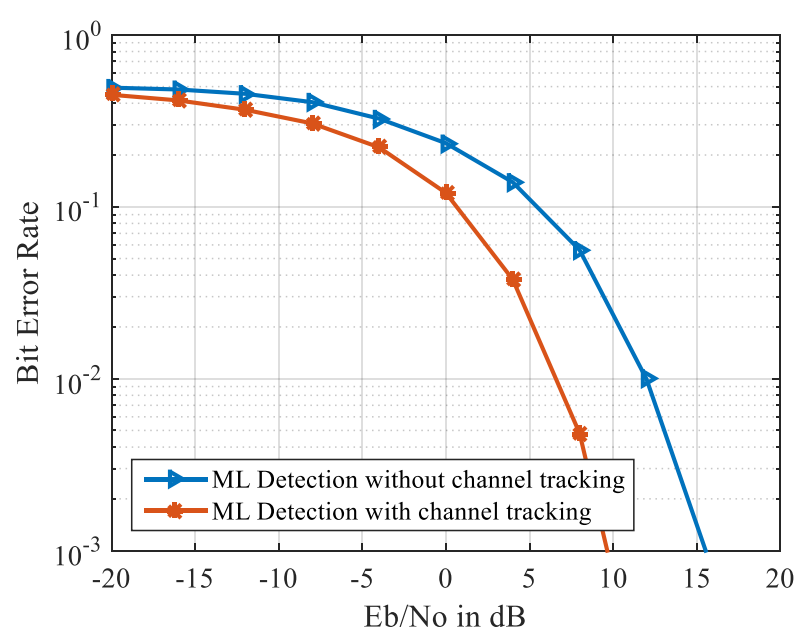

Figure. 5 Detection with and without the aid of channel information 
In Fig. 5, the BER of $10^{-3}$ has been achieved with $E_{b} / N_{o}$ of approximately $10 \mathrm{~dB}$ when channel information is tracked at the receiver. At the same time, $E_{b} / N_{o}$ needs to be approximately $15 \mathrm{~dB}$ to reach the same BER when channel state information is not available at the receiver. It shows that, error performance of the system can be improved with the aid of channel tracking algorithms.

The ML detector error performance in different fading scenario has been simulated and the results are presented in Fig. 6. For channel tracking RMMSE algorithm has been used. In both low fading and high fading environment, to attain the BER of $10^{-3}$, approximately $E_{b} / N_{o}$ of $15 \mathrm{~dB}$ is required. It has been verified that, the ML detector gives optimum performance in all fading environment including severe environments.

The performance of MIMO system for 4 number of transmitting and 4 number of receiving antennas is shown in Fig. 7.

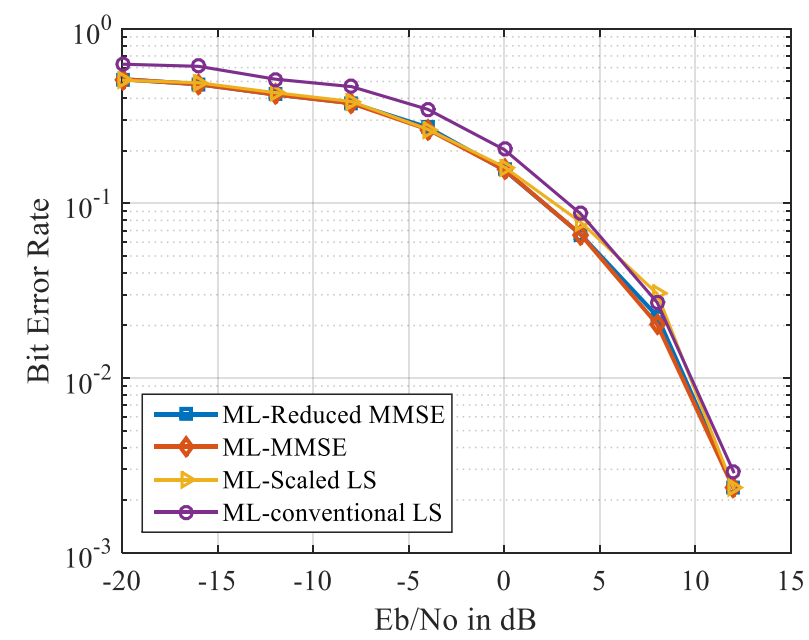

(a)

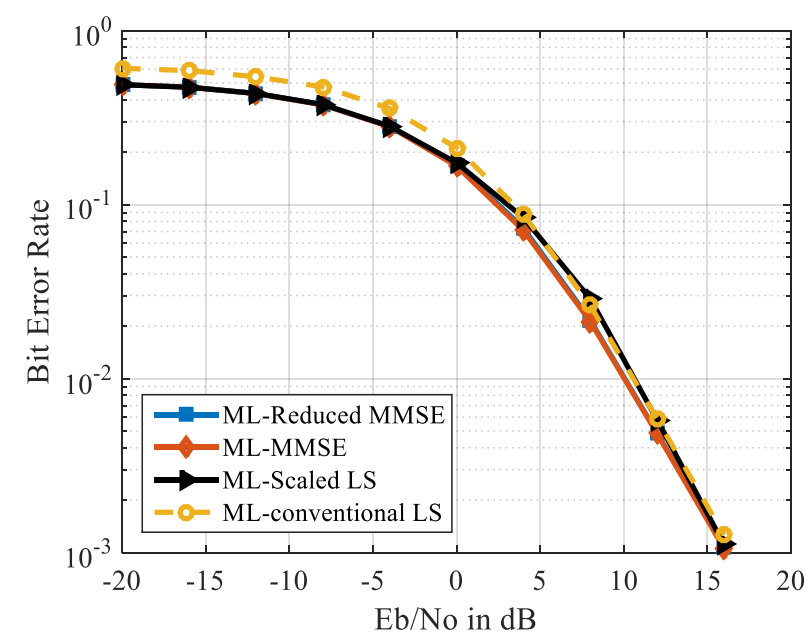

(b)

Figure. $6 \mathrm{ML}$ detector with various channel estimators over (a) SUI-1 (b) SUI-5

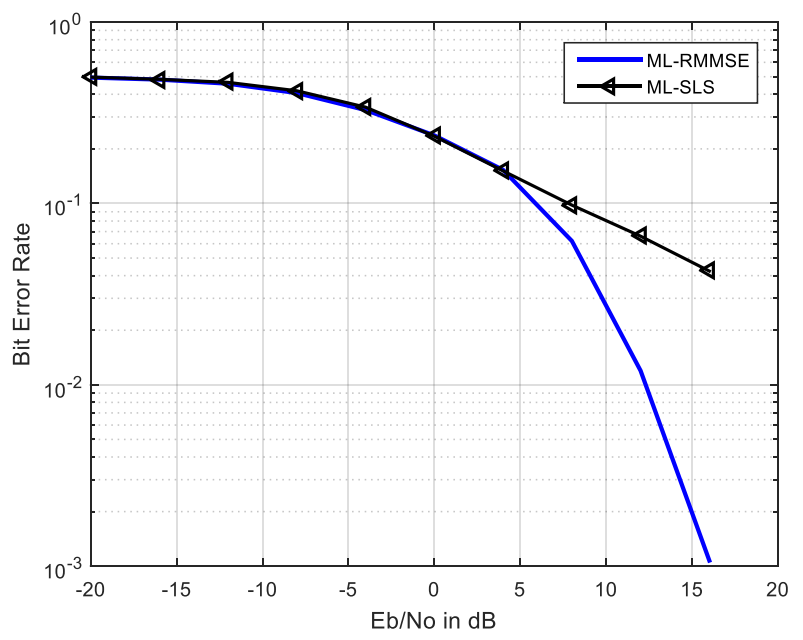

Figure. $7 \mathrm{ML}$ detector with various channel estimators over SUI-1 with $4 \times 4$ array structure

When the number of antennas is increased at the transmitting and receiving end, higher amount of signal energy is required to meet the same error rate regardless of the kind of detector and channel estimator used as shown in Fig. 7. The BER of $10^{-3}$ has been achieved with $E_{b} / N_{o}$ of approximately 15 $\mathrm{dB}$ when $2 \times 2$ antennas array is used. At the same time, $E_{b} / N_{o}$ needs to be approximately $16.5 \mathrm{~dB}$ to reach the same BER when the number of antennas is increased.

Finally, the BERs of the MIMO system are measured over different detection theory and shown in Fig. 8. The BER of $10^{-2}$ has been achieved with $10 \mathrm{~dB} E_{b} / N_{o}$ of approximately with ML detection algorithm but, $E_{b} / N_{o}$ needs to be approximately 15 $\mathrm{dB}$ to reach the same BER when the conventional MMSE detector is used. Also it has been observed that, ML with RMMSE techniques performs better than ML detection with conventional LS algorithm in all scenarios.

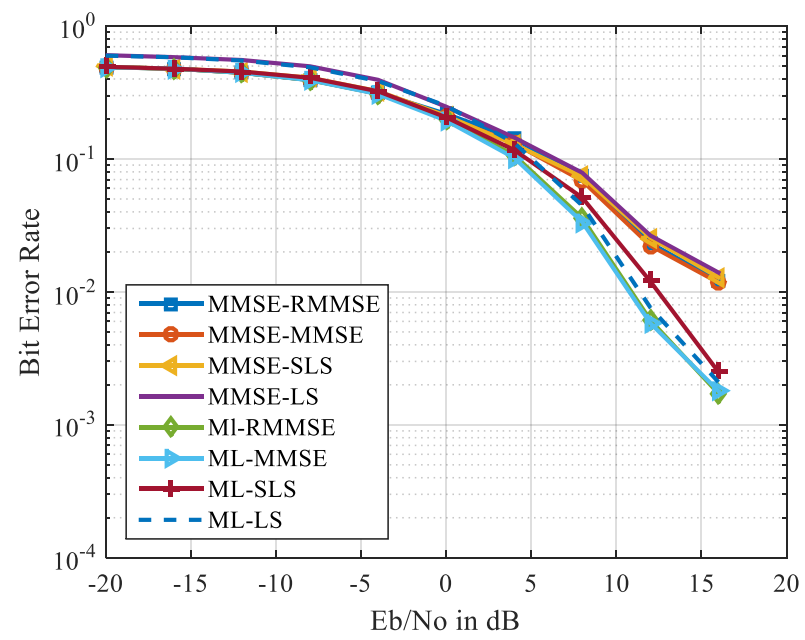

Figure. $8 \mathrm{ML}$ detector and MMSE detector Comparison under SUI-1 channel model 


\section{Conclusions}

The number of bit errors in the transmitted data sequence by including diversity gain in a WiMAX system has been analysed. Complexity reduced ML detection scheme can mitigate the ICI and ISI. The new performance evaluation results are presented in this paper with ML detection algorithm for different multi-antennae configurations. Application of various channel tracking algorithms indicate that Reduced Minimum Mean Square Error with ML detection provides good performance gain at low SNR values in WiMAX systems. The approximate SNR requirement to obtain the bit error rate of $10^{-2}$ is found to be $16 \mathrm{~dB}$ in MMSE based detector with RMMSE channel estimation and it is $10 \mathrm{~dB}$ in ML based detection with RMMSE channel estimation. However, in the signal detection scenario, since MMSE has limited performance and the ML detector is highly complex, research in the direction of sub optimal detection can be tried.

\section{References}

[1] M. Speth, S. A. Fechtel, G. Fock, and H. Meyr, "Optimum receiver design for wireless broadband systems using OFDM- Part1", IEEE Transactions

on communications, Vol.47,No.11, pp.1668$1677,1999$.

[2] A. Paulraj, R. Nabar, and D. Gore, "Introduction to space-time wireless communications", Cambridge university press, 2003.

[3] K. P. Bagadi, and S. Das, "Neural networkbased multiuser detection for SDMA-OFDM system over IEEE $802.11 \mathrm{n}$ indoor wireless local area network channel models", International Journal of Electronics, Vol. 100, No. 10, pp. 1332-1347, 2013.

[4] K. P. Bagadi, and S. Das, "Neural networkbased adaptive multiuser detection schemes in SDMA-OFDM system for wireless application", Neural Computing and Applications, Vol. 23, No. 3, pp. 1071-1082, 2013.

[5] K. P. Bagadi, and S. Das, "Efficient complex radial basis function model for multiuser detection in a space division multiple access/multiple-input multiple-outputorthogonal frequency division multiplexing system", IET Communications, Vol. 7, No. 13, pp. 1394-1404, 2013.
[6] K. P. Bagadi, and S. Das, "Multiuser Detection in SDMA-OFDM Wireless Communication System Using Complex Multilayer Perceptron Neural Network", Wireless personal communications, Vol. 77, No. 1, pp. 21-39, 2014.

[7] F. Wang, A. Ghosh, C. Sankaran, and S. Benes, "WiMAX system performance with multiple transmit and multiple receive antennas", IEEE $65^{\text {th }}$ Vehicular Technology Conference, VTC2007-Spring, pp.2807-2811, 2007.

[8] M. Gidlund, "On Wimax performance with multiple antenna transmission", IEEE International Conference on Consumer Electronics, Digest of Technical Papers, pp.1-3, 2007.

[9] R. Marks, "A technical overview of the Wireless MAN air interface for broadband wireless access", IEEE C802 16-02, 2002.

[10] IEEE LAN/MAN Standards Committee, "IEEE Standard for local and metropolitan area networks Part 16: Air interface for fixed and mobile broadband wireless access systems amendment 2: Physical and medium access control layers for combined fixed and mobile operation in licensed bands and corrigendum 1", IEEE Std 802.16-2004/Cor 1-2005, 2006.

[11] C. E. Shannon, "A mathematical theory of communication", The bell System technical Journal, Vol. 27, pp. 379-423, 623-656, 1948.

[12] F. Gao, and A. Nallanathan, "Blind channel estimation for MIMO OFDM system via nonredundant linear precoding", IEEE Trans. on Signal Process., vol. 55, pp. 784 - 789, 2007.

[13] C. C. Tu, and B. Champagne, "Blind recursive subspace-based identification of time-varying wideband MIMO channels", IEEE Trans. on Veh. Technol., vol. 61, pp. 662-674, 2012.

[14] J. Zhang, L. Hanzo, and X. Mu, “Joint decision directed channel and noise variance estimation for MIMO OFDM/SDMA systems based on expectation conditional maximization", IEEE Tans. on Veh. Technol., vol. 60, pp. 2139-2151, 2011.

[15] X. Lu, J. Xu, and G. Lin, "Channel estimation techniques based on short preamble and pilot MIMO-OFDM systems", Inter. J. of Dig. Cont. Technol. and its Appl., vol. 6, pp. 449-456, 2012.

[16] M. Biguesh, and A. B. Gershman, "Trainingbased MIMO channel estimation: a study of estimator tradeoffs and optimal training 
signals" IEEE transactions on signal processing, Vol.54, No.3, pp.884-893, 2006.

[17] K. P. Bagadi, and S. Das, "Minimum symbol error rate multiuser detection using an effective invasive weed optimization for MIMO/SDMA-OFDM system", International Journal of Communication Systems, Vol. 27, no. 12, pp. 3837-3854, 2014.

[18] K. P. Bagadi, V. Annepu, and S. Das, "Recent trends in multiuser detection techniques for SDMA-OFDM communication system", Physical Communication, Vol. 20, pp. 93-108, 2016.

[19] D. A. Shnidman, "A generalized Nyquist criterion and an optimum linear receiver for a pulse modulation system", Bell System Technical Journal, Vol. 46, No.9, pp.21632177, 1967.

[20] H. Jafarkhani," Space-time coding: theory and practice", Cambridge university press, 2005.

[21] L. Hanzo, J. Akhtman, M. Jiang, and L. Wang," MIMO-OFDM for LTE, WiFi and WiMAX: Coherent versus non-coherent and cooperative turbo transceivers", Vol. 9, John Wiley \& Sons, 2010.

[22] W. V. Etten, "An optimum linear receiver for multiple channel digital transmission systems", IEEE Transactions on Communications, Vol.23, No.8, pp. 828-834, 1976.

[23] G. J. Foschini, "Layered space-time architecture for wireless communication in a fading environment when using multi-element antennas", Bell labs technical journal, Vol.1, No.2, pp.41-59, 1996.

[24] P. W. Wolniansky, G. J. Foschini, G. D. Golden, and R. A. Valenzuela "V-BLAST: An architecture for realizing very high data rates over the rich-scattering wireless channel", International Symposium on Signals, Systems and Electronics, pp.295-300, 1998.

[25] G.D. Golden, C.J. Foschini, R.A. Valenzuela, and P.W. Wolniansky, "Detection algorithm and initial laboratory results using V-BLAST space-time communication architecture", Electronics letters, Vol. 35, No.1, pp.14-16,1999.

[26] S. Yang, and L. Hanzo, "Fifty years of MIMO detection: The road to large-scale MIMOs", IEEE Communications Surveys \& Tutorials, Vol. 17, No.4, pp.1941-1988, 2015.
[27] W. Van Etten, "Maximum likelihood receiver for multiple channel transmission systems", IEEE Transactions on Communication, Vol. 24, No.2, 276-283, 1976.

[28] A. Septimus, Y. Keller, and I. Bergel, "A spectral approach to inter-carrier interference mitigation in OFDM systems", IEEE Transactions on Communication, Vol. 62, No. 8, pp. 2802-2811, 2014.

[29] J.Z. Zhou, J.Y. Qin, and Y.C. Wu, "Variational inference-based joint interference mitigation and OFDM equalization under high mobility", IEEE Signal Processing Letters, Vol. 22, No. 11, pp. 1970-1974, 2015.

[30] V. Erceg, K. V. S. Hari, and M. S. Smith, "Channel Models for Fixed Wireless Applications", IEEE 802, 16.3 c-01/29rl, IEEE P802 16, 2001. 